\title{
The Design Study on Electrical Initiation Control Systems
}

\author{
Liang Wang (Corresponding author) \\ Engineering Institute of Corps of Engineers, PLA Univ. of Sci. \& Tech \\ No. 1, Haifuxiang, Nanjing 210007, China
}

Tel: 86-189-3603-5865 E-mail: qianxueningsi@163.com

Yaohua Wang

Engineering Institute of Corps of Engineers, PLA Univ. of Sci. \& Tech

No. 1, Haifuxiang, Nanjing 210007, China

Tel: 86-25-8082-0028Ｅ-mail: qianxueningsi@yahoo.cn

Xiaoqiang Yang

Engineering Institute of Corps of Engineers, PLA Univ. of Sci. \& Tech

No. 1, Haifuxiang, Nanjing 210007, China

Tel: 86-138-1388-5481Ｅ-mail: qianxueningsi@yeah.net

Kai Cheng

Engineering Institute of Corps of Engineers, PLA Univ. of Sci. \& Tech

No. 1, Haifuxiang, Nanjing 210007, China

Tel: 86-135-0518-1953 E-mail: chengkai911@msn.com

Haishan Yang

Military Representative Office of the General Department of Armaments in Zhengzhou Area

No. 23, Eastern Rantun Road, Zhengzhou 450051, China

Tel: 86-139-3719-6083 E-mail: yang67568578@163.com

Baoguo Zhu

Military Representative Office of the General Department of Armaments in Tianjin Area

No. 7, Yuhong Road, Hebei District, Tianjin 300240, China

Tel: 86-133-8991-0860 E-mail: zhbg@163.com

Xinwei Ji

Engineering Institute of Corps of Engineers, PLA Univ. of Sci. \& Tech

No. 1, Haifuxiang, Nanjing 210007, China

Tel: 86-139-0516-4135 E-mail: jxw0127@yahoo.com.cn

$\begin{array}{lc}\text { Received: September 30, } 2011 & \text { Accepted: October 21, } 2011 \quad \text { Published: December 1, } 2011 \\ \text { doi:10.5539/mas.v5n6p106 } & \text { URL: http://dx.doi.org/10.5539/mas.v5n6p106 }\end{array}$

The research is financed by National Great Engineering. 


\begin{abstract}
The safety design requirements of electrical initiation control system were firstly analyzed, and then according to the electrical initiation control system signal flow and detonation theory, the overall technical program of the electrical initiation control system was proposed, at last that the safety design of the main control box of the electrical initiation control system hardware and software was completed.
\end{abstract}

Keywords: Electrical initiation control system, Safety design, Precise blasting support system, Hardware and software, Detonation

\title{
1. Safety Design Requirements
}

The electrical initiation control system which as the control part of the precise blasting support system is the basic function of precise blasting support system to complete tasks, but also the system control center. As the special work environment for the precision blasting support system, in the design of the electrical initiation control system, there is a high requirements on the integrity of functions, performance, safety and reliability for the electrical initiation control system. The detailed design requirements as follows:

\subsection{Functional requirements}

(1) The system detonate seven lines orderly in accordance with the set procedures, each initiation line interval control accurately;

(2) In case of emergency, the system can follow the procedures directly detonate the sixth and seventh lines, initiation time control accurately;

(3) The control system has the function of fault detection and indication, and detect system lines, power, control relay fault condition, display specific fault location and type, detonate orderly in accordance with procedures after the completion and adoption, or can not detonate;

(4) The control system has a mechanical safety switch, be sure to manually detonate, to prevent misuse.

\subsection{Environmental adaptability}

(1) Temperature: meet requirement of the ARJ21-700 aircraft temperature envelope, operating temperature $\left(-40^{\circ} \mathrm{C} \sim+85^{\circ} \mathrm{C}\right)$, storage temperature $\left(-50^{\circ} \mathrm{C} \sim+70^{\circ} \mathrm{C}\right)$, normal operation in the condition of temperature variation;

(2) Vibration: Comply with 135GD001 (A version) and RTCA DO-160E "Environmental Conditions and Test Procedures for Airborne Equipment" in the provisions of the vibration environment. The three axial resistances were functional tests, vibration testing and vibration testing of high value. After three consecutive cycle vibration, the fixed component should not be loose, shifting, part of the electrical connections should connect properly, detonating devices should be in good condition, and it can be complete the set action safely and reliability;

(3) Heat: the device should be at a temperature not lower than $40^{\circ} \mathrm{C}$, relative humidity is not less than $95 \%$ in the hot and humid environment.

\subsection{Reliability}

(1) In line with the reliability requirements GJB1130-91;

(2) System reliability: Not less than 0.999 in the case of lower confidence limit of $90 \%$;

(3) Non-mandatory trigger probability: Not higher than $10^{-3}$ in the case of lower confidence limit of $90 \%$.

\subsection{Electromagnetic compatibility requirements}

EMC should be able to meet the following requirements: Device should be able to resist the natural environment and surrounding equipment from electromagnetic interference, and the device itself can generate electromagnetic interference suppression to the extent permitted, to ensure that equipment intended electromagnetic environment (test in interior aircraft) in the normal work of system failure or error will not cause detonation. EMC minimum limits should be consistent with the relevant requirements of GJB151A; equipment, electromagnetic emission and susceptibility tests should meet the relevant requirements GJB152A: equipment grounding, bonding, and shielding requirements should be consistent with GJB1210; equipment, electromagnetic compatibility should be managed to the requirements of GJB/Z 17.

Equipments need to meet the GJB151A-1997 in the CE102, CE106, CS101, CS106, CS114, RE102 and RS103 (Note: CE conducted emissions, RE radiation emission, CS conduction sensitivity, RS radiation sensitivity).

\subsection{Power Requirements}

System power requirements are as follows: 
(1) The system has an independent power supply;

(2) In line with GJB367A-2001A of the provisions 3.28, can provide the system required power to self-test, control, and detonate;

(3) Power continuous working time is not less than 4 hours;

(4) The system can provide the energy required to detonate normally in the extreme environments.

\subsection{Structural requirements}

(1) The system structure should be in line with the technology agreement of "ARJ21-700 aircraft cabin air emergency pressure relief and service door open system", the control panel should be not larger than $200 \mathrm{~mm} \times$ $120 \mathrm{~mm} \times 80 \mathrm{~mm}$;

(2) The system weight: The total weight of system should be controlled in the $30 \mathrm{~kg}$ (without cable) or less.

\subsection{Other requirements}

(1) It is easy to control, and the control buttons should be with a shield and have eye-catching warning text;

(2) Normal system maintenance cycle should be less than 6 months;

(3) System storage period: The provision environmental conditions should be in less than 2 years.

\section{Overall Design}

According to the proposed security requirements on the electrical initiation control system of the accurate explosion support system of design task book, the first initiation of electrical control system of the overall design, the design follows the safe, reliable, simple, easy to use principles. Electrical initiation control system includes three main parts, they are control panels, main control box, and detonating lines, a total of seven initiating lines, two to the aircraft skin, for transfer initiation felt a pressure relief port on the energy required detonators, three initiating lines are used to transfer detonation to lift hinges and unlock constraints of the energy required insensitive detonators, another two transmission lines are used to produce an explosive power to open the door.

The control panel is the interface to control the precise blasting support system, as shown in Figure 1. Set a safe switch, the normal initiation button and the LED lights, emergency initiation button and LED indicator, LED indicator and fault normal LED indicator. Safe switch is used to prevent misuse of the system operator, the safe switch is not closed, and the system can not perform initiation operation. Normal detonation buttons and emergency buttons for initiating detonation were triggered by the normal and emergency initiation command, the fault indicator can display not only failed state, and the length of time can be represented by flashing fault code indicating the type of fault.

The main control box is the core component of the electrical initiation control system; its internal control circuit board can achieve timing of the precise explosion support system control and has fault detection capabilities, appearance shown in Figure 2. Surface normal light settings, fault indicator, power switch and electrical connectors. One normal and fault indicator lights corresponding indicator function with the same control panel. The main control box connected via electrical connector battery box, control panel and detonation circuits, control signals and initiation energy to complete the signal transmission. The main control box powered by a separate battery box, battery box and put together the main control box.

Initiation line refers to the control box and the initiation of the connection lines between components, complete the test signal and detonation energy of signal transmission. Blasting support systems based on accurate design, detonating a total of seven lines, $1 \#$ and $5 \#$ detonating line length of $12 \mathrm{~m}$ is responsible for lifting service constraints and the door hinge, 6 \# and 7 \# the length of enameled wire path $20 \mathrm{~m}$, is responsible for cutting relief mouth. Firstly, open the control box power, energy storage circuit of the RC pre-charging, self-test is carried out after charging, the charging circuit voltage, 7 lines and 7 detonating relays in order to perform self-test, if we find fault, through fault LED Indicator fault code indicating the type of fault and fault, suggesting that maintenance personnel to troubleshoot; when test results showed normal, the system enters standby mode. When the aircraft failed during flight, the flight personnel detonated the actual situation to decide whether to open up escape routes. When the need for initiation, first turn off safety switch, and then depending on the circumstances or the emergency button is pressed the normal detonation button to open up the flight crew escape tunnel, complete system works.

(1) Press the start button to start the emergency relief system; delay 15 seconds, cutting service door hinge plate and detonating the explosive cutting cord lock; further delay 2 seconds, to promote the service door of the power plant fire; 
(2) Press the start button, emergency, service door hinge cutting detonating the explosive cutting board and cable lock; Then delay one second, so promoting the service door of the power plant fire.

Program design elements are as follows:

(1) In order to improve security, reliability and ease of maintenance, power using a lithium battery-powered, the battery installed in a separate box, power supply for the entire electrical system, battery capacity is designed at $2300 \mathrm{mAh}$, the system can support more than 10 hours of continuous work;

(2) The initiation load more, detonating a small load resistance, initiation of high energy demand (particularly insensitive detonators, detonating the current needs of more than $3 \mathrm{~A}$ ), can not use the battery directly to detonate, so the energy use of capacitor discharge initiation instantaneous discharge manner;

(3) Safety switch on the control panel settings, control a safe-off relays, safety relays in the initiation of the main contacts in series circuit in the main, by the artificial manipulation, from security to confirm the role, when the safety switch turns off, unable to perform initiation actions to improve system security and reliability;

(4) Set fault detection circuit, when power lines in the system impedance, low resistance fault, energy storage circuit failure, power supply and relay fault detection, standby tank circuit voltage of the line and the detonation resistance testing and found that failure alarm, to ensure the system's high security requirements.

\section{Hardware Security Design}

The main control box is the control center of the entire system, the circuit takes the modular design, the chip-centric solution that is easy to achieve reliable and flexible control and fault detection needs, mainly by the seven parts components: a microprocessor circuit, power circuit, detonating circuit, fault detection circuit, the programming circuit, the circuit command buttons and LED display circuit.

The microprocessor is the "brain" of the entire circuit which is responsible for receiving initiation instruction, POST, fault detection; given the timing initiation signal, the receiver fault detection signal, and fault information display functions. Lithium battery power supply circuit to transform voltage DC12V, DC24V voltage, DC5V voltage, the microprocessor circuit, display circuit, self-test circuit and detonating circuit to provide power. Stage of development and programming circuit used to modify the programming and software debugging. CPU control circuit to receive the signal initiation through the drive circuit control relay to complete the electrical detonator ignition initiation. Command key input circuit according to flight personnel's instructions, enter a different initiation control signals to control the pressure relief port and the service door open way. LED display circuit through the light display system fault detection results, and indicates the fault location and type. Fault signal detection circuit voltage through the circuit related to the initiation signal amplification filtering, the input single-chip, after treatment failure through the display circuit displays the results. The following are the various sections.

\subsection{Microprocessor Circuit}

The microprocessor selected ATMEL Corporation Atmega16L, mainly on the following considerations (Zhang, Song Tao, et al, 2009, pp.415-426):

(1) The chip is based on a RISC (Reduced Instruction Set Computer, reduced instruction set computer) architecture instructions 8-bit microcontroller, running speed, low power consumption for battery-powered systems;

(2) The Harvard bus architecture, separate program memory and data memory, CPU direct access to all program and data memory, system security, high reliability;

(3) Chip voltage is $2.7 \mathrm{~V} \sim 5.5 \mathrm{~V}$, wide operating voltage range, anti-jamming;

(4) Chip integrated circuit debugging unit, can be achieved online through the JTAG debug and download capabilities, can make the AVR microcontroller into a high flexibility to meet the diverse needs of high-speed microprocessor;

(5) High drive capacity with 16 programmable I/O port can directly drive a large load current, the port also has programmable internal pull-up resistor can be used for man-machine control instruction key input, output and fault control signal initiation signal display;

(6) With 8 built-in 10-bit ADC, to meet the needs of fault detection signal voltage without the need for external AD conversion, simplifying the circuit structure;

(7) Has an internal clock source, the program uses the internal clock, simplifying circuit structure and improve the system security and reliability. 
Circuit R18 and C10 are single-chip hardware reset circuit, instantaneous power, the RESET pin is pulled low, single-chip program memory from the beginning of the program is running, then the voltage charging capacitor $\mathrm{C} 10$ to rise exponentially high, the device enters the normal operating state.

P5 standard JTAG port is a single-chip emulation debug interface, using simple four-wire structure: TMS, TCK, TDI, TDO, were selected for the test mode, test clock, test data input and output test data, enabling the endpoint trace debug the program and download updates, easy software debugging and program updates.

Inductor L1 capacitor C9 to form the power LC filter circuit, the MCU internal analog-digital conversion modules to provide clean power to improve anti-interference ability and analog to digital conversion accuracy.

In addition, when the MCU pin assignments, the strong signal and weak signal separation, separate input and output signals, to improve anti-jamming capability, PD2, PD3 pin for normal initiation and detonation button emergency signal input detection, PB0 PB6 for 1 to 7 lines of detonating detonation signal output, PA0 PA6 for 1 to 7 detonating line fault detection of analog signal input, Q9B and Q10B for normal and fault indicator output.

\subsection{Power Circuit}

Power circuit design is very important, the higher requirements for the whole high-security, high reliability of the power of, the need to consider the load on the power supply voltage, capacity, quality, ripple, temperature characteristics and other performance requirements related to the circuit job security and reliability. In view of the system independent of portability and power requirements, according to the detonation circuit of power demand and standby time and energy security requirements, the machine uses lithium battery-powered, given the Li-ion battery with lower voltage and temperature will be significantly reduced capacity characteristics, to meet the whole working temperature $-40^{\circ} \mathrm{C} \sim 85^{\circ} \mathrm{C}$ need to take full account of the battery capacity margin.(Liu Cheng, Zhao.2009, pp.15-16)(Ghaderi A, Sanada A, Nassiraei Amir AF, Ishii K, Godler I. 2008, pp.26-29).

Power circuit uses four lithium battery powered lithium batteries in series; each battery has a capacity of $2300 \mathrm{mAh}$, the total supply voltage of $14.4 \mathrm{~V}$. As with the use of power, the voltage will change, in order to make the circuit power supply is sTable, reliable, to minimize the ripple, noise interference, the output voltage lithium battery pack and DC/DC2 through DC/DC1 transform voltage $12 \mathrm{~V}$ and $24 \mathrm{~V}$ voltage, $36 \mathrm{~V}$ DC voltage obtained in series to meet the detonation initiation voltage of the load demand, $12 \mathrm{~V}$ output voltage through the LM7805 circuit sTable, transformed into a sTable DC voltage of $5 \mathrm{~V}$, the supply of single-chip circuits. Power circuit diagram shown in Figure 3.

DC1 and DC2 respectively MORNSUN WRB1224YMD and WRB1212YMD, the power module input power and output power isolation, isolation voltage $1500 \mathrm{VDC}$, while using a wide supply voltage input technology, the input range up to $9 \sim 18 \mathrm{~V}$, can expand the effective battery voltage range, up to $85 \%$ efficiency, with short circuit support self-recovery, the normal operating temperature range $-40^{\circ} \mathrm{C} \sim+85^{\circ} \mathrm{C}$, shielded metal package, mean time between failures MTBF $>1000000$ hours, anti-lightning surge to meet the three above The module can meet the system power supply voltage, capacity, temperature and security, reliability and other requirements.

As the circuit 9, due to DC1 and DC2 power module requirements, output load is not less than $10 \%$ of full load, so the output connected to the resistors R1 and R2, select the appropriate resistance and power to the DC output power is always greater than $10 \%$. To further meet the requirements of reducing the ripple in the output and input connected to the filter capacitor $\mathrm{C} 1, \mathrm{C} 2, \mathrm{C} 3, \mathrm{C} 4$, and joined the $\mathrm{C} 5, \mathrm{C} 6$ to improve high frequency characteristics.

\subsection{Detonation Circuit}

Detonation circuit is shown in Figure 4, the main function is to receive the microprocessor initiation signal, security, stability, and reliable energy storage circuit connected with electric detonators, detonating both safe and reliable, but also reliable when placed in standby safety state, the circuit including the discharge areas, storage areas, detonation control and safety circuits, and several other important part of the detonator to detonate the load.

\section{Design features are:}

(1) Since each electric detonators and detonating the safety of electrical ignition current of $5 \mathrm{~A}$, the resistance only, he felt a porTable power source is difficult to achieve electric detonators and felt a direct electrical ignition detonation, so the use of the RC Chu to discharge an indirect way to achieve safe, reliable initiation;

(2) Taking into account the existence of electronic switching elements of the security breakdown, detonating switch element selected HF Company HF-115/1 Form C type SPDT relay to meet the detonation control and fault detection needs. Maximum breaking current of 16A, maximum voltage of breaking $125 \mathrm{VDC}, 5000 \mathrm{~V}$ 
between coil and shock isolation capabilities, in line with IEC 60335-1 standard, adaptation to the environment temperature of $-40^{\circ} \mathrm{C} \sim 85^{\circ} \mathrm{C}$, humidity of $35 \%$ to meet the $85 \%$ to meet the detonation control requirements, the relay passed temperature and vibration test machine, that it can meet the system's security requirements.

(3) In order to improve circuit performance, achieve security, the need for reliable detonation, detonation voltage selector to DC 36V, see "Power circuit" part;

(4) The charging resistor $\mathrm{R} 15$ design selected for the $1.8 \mathrm{k} \Omega$, maximum power is $\mathrm{P}=\mathrm{i}^{2} \mathrm{R}=0.72 \mathrm{~W}$, the maximum charge current is $36 \mathrm{~V} / 1.8 \mathrm{k} \Omega=20 \mathrm{~mA}$, resistor power rating selected $2 \mathrm{~W}, 7$ initiation maximum charge current of the line is $140 \mathrm{~mA}$, so the power DC-DC module WRB1224YMD is rated for 250mA, WRB1212YMD rated current of $500 \mathrm{~mA}$, charging and microprocessors to meet the demand for electricity;

Design capacity of the storage capacitor C3 is very important, too small to achieve reliable detonators, detonating the load requirements, much increased by the volume of unnecessary and dangerous. There needs to be accurately calculated according to the load, accurate burst support system in order to ensure safe, reliable initiation, initiation of each line with four electric detonators, detonating series-parallel connection method is shown in Figure 5.

Since the source of initiation for each detonator initiation resistance of $2 \Omega$, because each current channel, the total resistance of detonation is still $2 \Omega$ of electric detonators, detonating $5 \mathrm{~A}$ (the duration must be greater than $1 \mathrm{~ms}$ ), thus requiring a large total current should be detonated at $10 \mathrm{~A}$, each detonator in a $1 \mathrm{~ms}$ time required to obtain the initiation energy:

$$
W=I^{2} R T=5^{2} \times 2 \times 1 \times 10^{-3} \mathrm{~J}=0.05 \mathrm{~J}
$$

Detonation, the energy storage capacitor to discharge to the detonator, the discharge current is $i=(36 \mathrm{~V} / 2.5 \Omega) \cdot e^{-\left(t / 25^{*} \mathrm{C}\right)}$ (considering $0.5 \Omega$ line resistance), in order to ensure the close of current is $1 \mathrm{~ms}$, each detonator can guarantee $5 \mathrm{~A}$ current work, the formula becomes to $2 \times 5<(36 / 2.5) \cdot e^{-\left(1 \mathrm{~ms} / 25^{*} \mathrm{C}\right)}$, the calculated capacitance $\mathrm{C}$, so the case in the left margin, select the $2200 \mu \mathrm{F}$ should be greater than $1097 \mu \mathrm{F}$ capacitor energy storage capacity, this time, the initiation of each resistor to obtain energy:

$$
W=0.5 \int_{0}^{1 m s}\left[(36 / 2.5) \cdot e^{-\left(t / 2.5 \times 1500 \times 10^{-6}\right)}\right] \times 2.5 d t=0.2 J>0.05 J
$$

Meet the requirements of reliable detonation.

(5) System boot time required for charging is $T_{c}=3 \tau=3 R C=3 \times 1.8 \mathrm{k} \times 1500 \times 10^{-6}=8.1 \mathrm{~s}$.

(6) Discharge circuit is the role of system shutdown, it can within the time specified in the charge capacitor all released, to ensure that no power storage devices to meet security needs. Voltage on the capacitor decreased exponentially according to:

$$
U(t)=U_{0} \times e^{-t / R C}
$$

Where is the initial capacitor voltage, is a natural constant. Generally believed that after the voltage down to $0.7 \%$ of original value, that the basic end of the discharge, then the required time of 10 seconds are:

$$
T=5 R C=5 \times R \times 1500 \times 10^{-6} \times 7=10 \mathrm{~s}
$$

Calculated to be $\mathrm{R} \approx 190476 \Omega$, the circuit selected high-precision conventional resistance $150 \mathrm{k} \Omega$, the discharge time of $7.9 \mathrm{~s}$; the system can meet the design requirements.

(7) In order to achieve the microprocessor $\mathrm{I} / \mathrm{O}$ port on the initiation of control relay, added the current amplification drive components NPN transistor Q5, diode D8 in the transistor turn-off instant release out of the relay coil of the induced electromotive force to protect circuit;

In order to improve system security and prevent the misuse or critical component failure, malfunction detonators, detonating the main back on the road to join in by the safety switch control relays, safety switches need manual confirmation.

\subsection{Key Input Circuit}

In order to improve the system of anti-jamming capability, the switch input for transmission using optical coupler, isolated input signal interference. (Yang Xue, 2006, pp.41-42). The advantage is one-way signal transmission, the input and output full realization of the electrical isolation, the output signal has no effect on the input side, has a strong common-mode rejection, anti-interference ability, sTable, non-contact, service life long, high transmission efficiency. The circuit design as shown in Figure 6.

The circuit used a nonlinear TLP521-2 dual-input dual-output coupler, the coupler current transfer characteristic 
curve is nonlinear, switching signals for transmission, the operating temperature of $-55 \sim 100^{\circ} \mathrm{C}$. TLP521 work prior to the forward current $16 \mathrm{~mA}$, due button trigger switch is $\mathrm{DC} 12 \mathrm{~V}, \mathrm{R}=(12-1.5) / 16 \approx 0.656 \Omega$, the collector current is typically $1 \mathrm{~mA}$, so $\mathrm{R} 8$ resistor selected $620 \Omega$, design $\mathrm{R} 9=4.7 \mathrm{k} \Omega$.

\subsection{Fault Detection Circuit}

Fault detection is the main control box must have important functions in standby power and the power, energy storage circuit, detonating line fault detection, fault is found the alarm, it is important to ensure reliability. Fault detection circuit is designed around the detonation circuit, the circuit including detection of incentives, auxiliary testing, measurement and control and signal amplifier. Excitation circuit which detects the positive voltage from the $36 \mathrm{~V}$ via a current limiting resistor R4 and the detection and control relay normally closed contact connected to one end while the load is connected with the signal amplification circuit, the output signal and the signal amplifier circuit connected to the microprocessor assisted detection of Atmega16 ADC I/O port.

Through the auxiliary detection circuit can be realized on the detection of stored energy storage circuit, instantaneous power, the microprocessor detects the auxiliary detection output voltage, which is the brightest capacitor charging current and the resistor R5 and the product, with the end of charge, the voltage gradually to zero, start charging the most, about $1.4 \mathrm{~V}$, the voltage through the detection can be realized on the energy storage circuit fault (including the charging voltage is too low, capacitor leakage and other faults) test.

Provide incentives for the detection circuit detects a excitation voltage, current, when the detection relay (the limiting resistor R4 resistance selected is $3.6 \mathrm{k} \Omega$ ) is not open, the resistance and the load line, load, detonators in series relationship, this flow detection when the detection current is about detonators $10 \mathrm{~mA}$, well below the safety detonator initiation current safety testing can be achieved, then detonate the line voltage of about $0.02 \mathrm{~V}$, the signal amplification circuit consists of a precision operational amplifier with integrated to the proportion of operational amplifier designed for 82 magnification, the signal amplification circuit the normal output voltage of $1.64 \mathrm{~V}$, just a microprocessor analog-digital conversion input signal range of intermediate values, the highest detection accuracy. If the initiation time of poor contact or short-circuit line, line resistances will amplifier output voltage will deviate from deviate from the normal $2.2 \Omega$, to detect the line impedance and low resistance faults.

If the boot, the error is on safety switch, the signal amplifier input voltage up to $36 \mathrm{~V}$, the signal will be accurately detected the microprocessor analog-digital conversion, and the police, to achieve safety switch on the power failure detection. In order to protect the $36 \mathrm{~V}$ voltage input amplifier circuit integrated operational amplifier, the amplifier input access to a $1.2 \mathrm{~V}$ regulator diodes.

In order to achieve control of initiation is normal movement detection circuit, power-fault self-test, the microprocessor control circuit in turn closes the brightest initiation, the normally open relay switch contacts together, this time under normal circumstances, amplifier circuit detection voltage should be $0 \mathrm{~V}$, or explain the relay error.

One of the auxiliary energy storage circuit detection circuits for fault detection and fault detection of the line impedance is the case in static fault detection system for static testing, test, compared with the action of the relay dynamic testing.

In a microcontroller as the core instrument, the need for the collected signals between analog and digital conversion, and the microcontroller can handle these signals, the signal is converted by the A/D converter to complete. A/D converter according to the different works can be divided into two categories: direct type A/D converter and the indirect type A/D converter. Direct converter work is the input analog signals into digital signals directly, without intermediate amount of change; indirect converter work, you need to input analog signals into the middle of volume, such as time, frequency, pulse width and so on, and then converted to digital signals. At present the application of more indirect type A/D converter has three types: successive approximation, dual integral and V/F transform. A/D converter output signal according to the different accuracy of significant digits can be divided into four, six, eight, 10 and many other types.

The fault diagnosis system has ATmega16L, low-power 8-way internal MCU, serial output, and 10-bit successive comparison of the A/D converter (ADC). A/D converter with an 8-channel analog multiplexer connected to PA from 8-port single-ended input voltage is sampled. A/D converter reference voltage analog power supply option, the external AREF pin inputs and an internal $2.56 \mathrm{~V}$ reference voltage is input through the gain amplifier before converting to digital data. A/D converter by "ADC Multiplexer Selection Register-ADMUX", "ADC Control and Status Register A-ADCSRA" control, transformation results in the "ADC data register --ADCH/ADCL" read.

Detection signal A/D converter reference voltage selected sTable internal reference voltage single-chip 2.56V, 
the chip port input current by sampling rate, input voltage and other factors. Of these factors, the signal source impedance and the chip operating modes do not consider, lies in the sampling rate and input voltage.(Don Juan, Wang Wendi, Lv Changfei, 2007, pp.138-140)(Tsmoto S, Tanaka H. Primerose, 1995, pp.389-405)(B. Walczak, DLMassart, 1999, pp.1-16).

In the op-amp circuit design, operational amplifier OPA335 role not only on the input signal amplification, more important is the sampled signal with a continuous output port of the microcontroller ADC A/D conversion of data between the sample buffer, because the internal MCU A/D converter is 8-channel analog multiplexer, the impact of rapid data acquisition A/D conversion speed and accuracy; the other side of the PA signal and the sensor output signal common mode rejection, to avoid noise and other sensing spurious effect of noise on the signal, the input voltage to ensure safe, reliable and sTable.

\section{Software Safety Design}

\subsection{Software Development Environment}

Outstanding performance based on the AVR microcontroller, many companies have introduced the use of high-level language programming development environment, makes the MCU software development more targeted. ATMEL's AVR Studio software is developed using the AVR microcontroller to provide a free integrated development environment, including assembly language and $\mathrm{C}$ language, has the following characteristics ( $\mathrm{Li}$ Xun, et al, 2002, pp.25-26).

(1) Support AVR assembler editor, compile, link and generate object code;

(2) Embedded AVR GCC high-level language interface;

(3) Containing AVR software simulator;

(4) Simulation debugging platform can also meet the ATMEL Corporation designs introduced a special simulator, such as real-time on-board simulator ICE40, ICE50, real-time on-chip emulator JTAG ICE, JTAG ICE mk II, in order to achieve the system's line of hardware emulation debug features and object code downloads.

Accurate burst support system software AVR Studio 4.13SP2 + WinAVR (provide the C language compiler) for design and development.

\subsection{Software Design}

Accurate burst control software support system must first be able to achieve the function of the system, including the exact start blasting security system, the system's accurate movements, burst support system for accurate monitoring of working conditions, ready to diagnose possible problems and promptly issued a warning, ensure system security, stability and reliability.

Secondly, the software should have some anti-jamming capability. Blasting support systems accurately the complexity of the work environment requiring a high anti-jamming capability and reliability, anti-jamming software design is a way to ensure system reliability, the system can solve some of the hardware design can not solve the problem and prevent the external interference caused by the system control failure. In the design we use a variety of techniques, such as the use of digital filtering technology acquisition signal processing to ensure accuracy of the measured signal; signal denounce algorithms using keys, time redundancy, the signal from external noise, to ensure the security of key inputs, reliability; in the program set software watchdog technique, to prevent the program "run off", time to restart the program. The anti-jamming technology can improve the stability of the system is running, to ensure accurate burst support system to achieve the function of normal.( Yanlei Qin, Yushi Lu, Juan Wang, et al, 2006, pp. 69-78).

Third, the system programs various functions clear, easy to modify maintenance. Accurate burst support system control software written using top-down approach, each part of the modular design, the system starts, self-test, monitoring, initiation and other functions into multiple modules, and between the flags by setting the variable to transmit information. Accurate burst support system using this method to write software, not only to improve the readability of the program, but also to modify the test program, the system software to ensure security and reliability.

\subsection{Software Processes and Functions}

Initialized by the system control software program, automatic testing procedures, data collection procedures, data, testing procedures, data processing procedures and algorithms, the interrupt service routine and so on.

When the system and did not complete self-test fails, the normal indicator light flashes, the system enters standby mode, then the system continuously detects detonation signal input information. When the input signals, 
the system determines the type of signal firstly, and detonates the normal timing of initiation and completion of emergency action initiation.

\section{Conclusions}

In this article, the safety design of the main control box of the electrical initiation control system hardware and software was completed. Hardware design, including microprocessor circuits, power supply circuits, detonating circuits, key signal input circuits and fault detection circuits. Software design, including system initialization, self-test program, data acquisition, data testing procedures, data processing procedures and algorithms, interrupt service routine, etc, in which the system self-test program, including static testing and dynamic testing.

\section{References}

Don, J., Wang, W. D, Lv, C. F. (2007). Based on New AVR Microcontroller Greenhouse Control System. Micro-computer Information. 138-140.

Ghaderi, A., Sanada, A., Nassiraei Amir, A. F., Ishii, K., \& Godler, I. (2008). Power and Propulsion Systems Design for an Autonomous Omni-directional Mobile Robot. Applied Power Electronics Conference and Exposition, 26-29. http://dx.doi.org/10.1109/APEC.2008.4522732

Li, X., et al. (2002). AVR Microcontroller Application Technology. Beijing: Beijing Aerospace University Press, 25-26.

Qin, Y. L., Lu, Y. S., Wang, J., et al. (2006). Comparison Study of System Safety Analysis Method. Journal of Safety Science and Technology, 2, 69-78.

Tsmoto, S., Tanaka, H. P. (1995). Probabilistic Rule Induction Method Based on Rough Sets and Resampling Methods. Computational Intelligence, 389-405. http://dx.doi.org/10.1111/j.1467-8640.1995.tb00040.x

Walczak, B., Massart, D. L. (1999). Rough Sets Theory. Chemo metrics and Intelligent Laboratory Systems, 1-16. http://dx.doi.org/10.1016/S0169-7439(98)00200-7

Xue, Y. (2006). The Development CO Gas Concentration Monitor Based on Short Message. Harbin: Harbin Institute of Technology master's thesis, 41-42.

Zhao, L. C. (2009). Intelligent Mobile Robot Power Module Design and Implementation. Instrumentation Technology, 15-16.

Zhang, S. T., et al. (2009). AVR Microcontroller C Programming Language Design Essence. Beijing: Electronic Industry Press, 415-426.

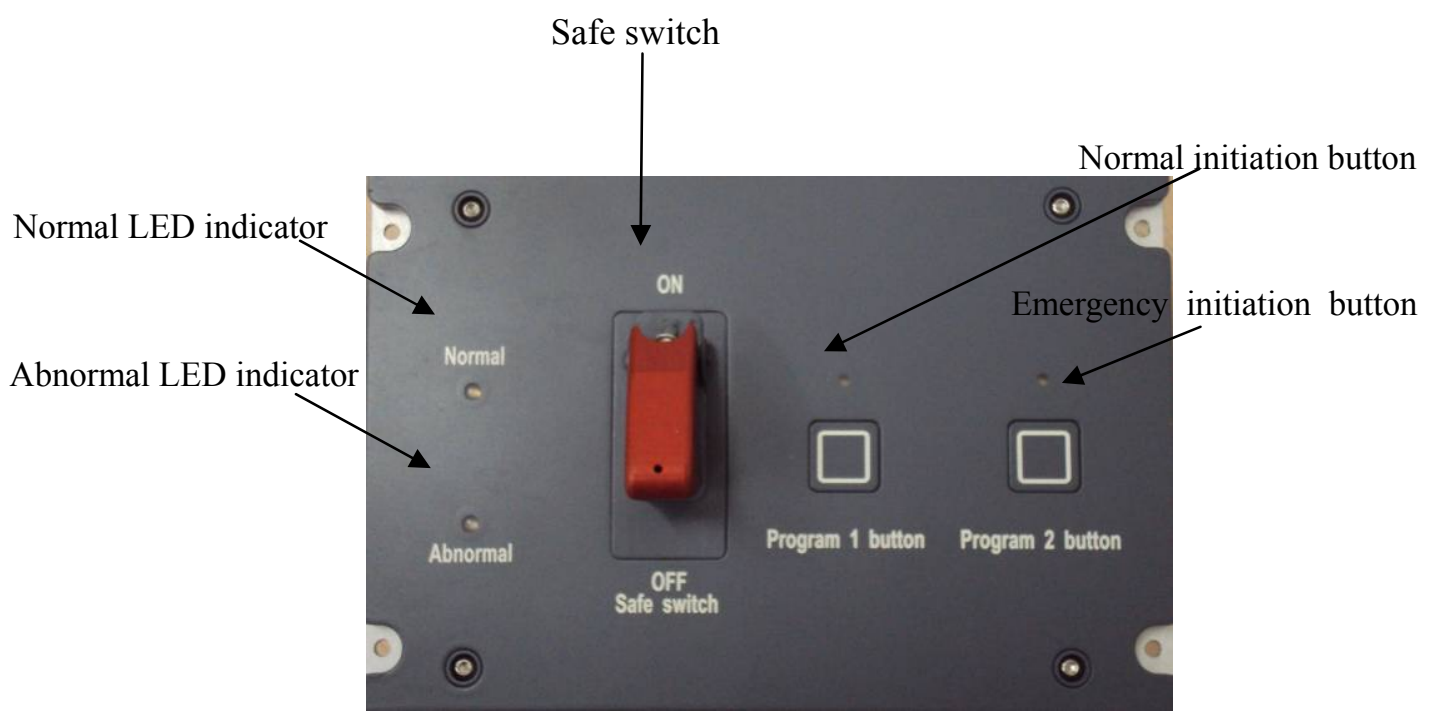

Figure 1. Control Panel 


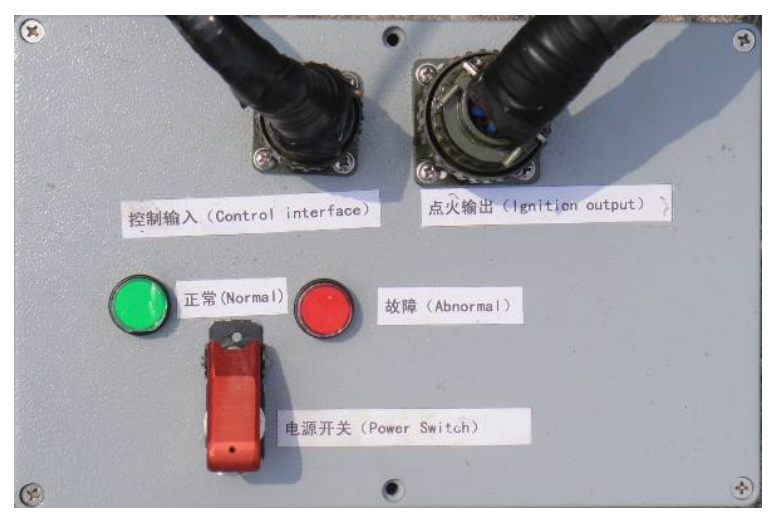

Figure 2. Main Control Box

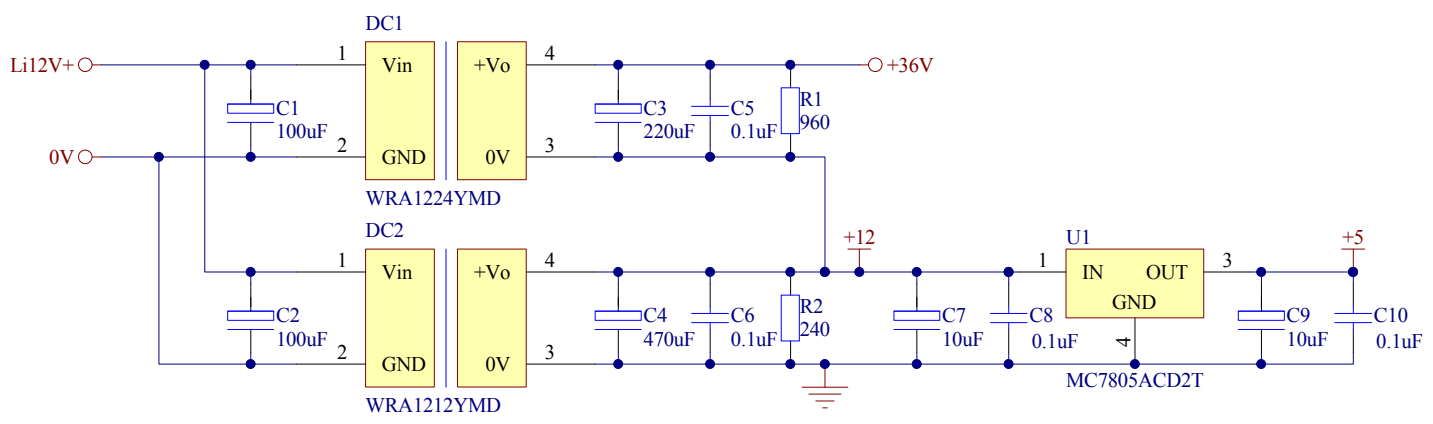

Figure 3. Power circuit schematic

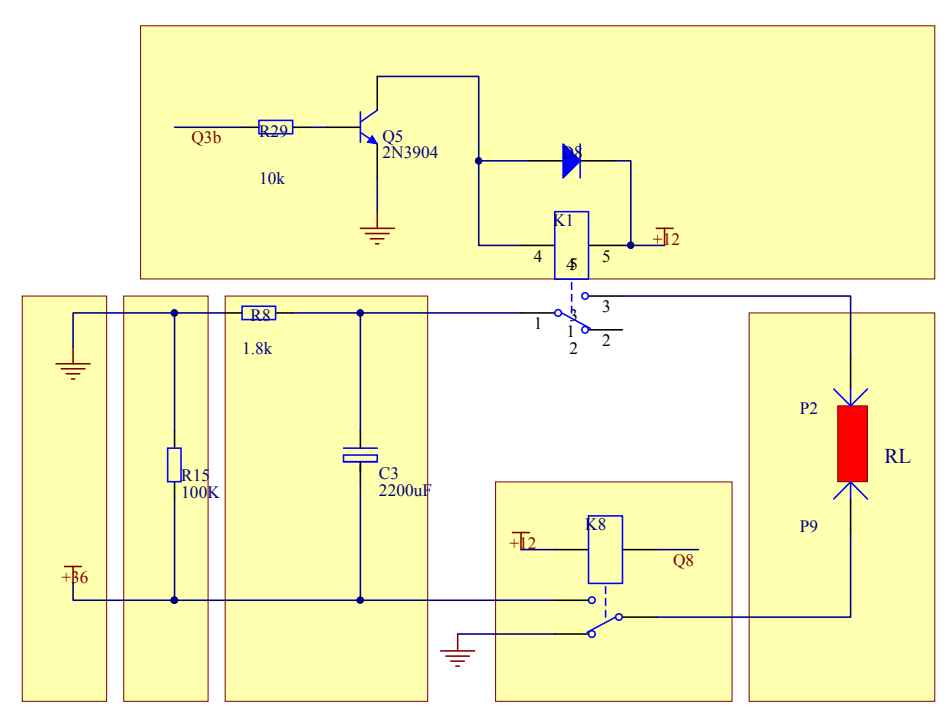

Figure 4. Detonating circuit diagrams 


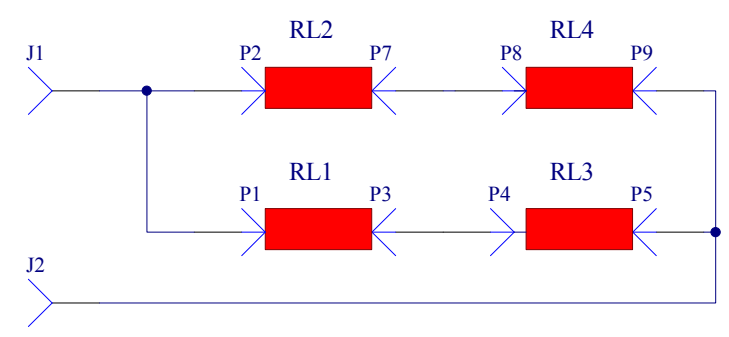

Figure 5. Connection electric detonators

\begin{tabular}{|c|c|c|c|c|c|}
\hline \multirow[b]{2}{*}{ DIla } & \multirow[b]{2}{*}{ R8 } & \multicolumn{2}{|c|}{ U2 } & \multirow[b]{2}{*}{ DI1b } & \multirow[b]{2}{*}{ R9 } \\
\hline & & 1 & 8 & & \\
\hline & 620 & 2 & 7 & & $4 \mathrm{~K} 7$ \\
\hline DI2a & R10 & 3 & 6 & DI $2 b$ & R11 \\
\hline & 620 & 4 & 5 & 11 & $4 \mathrm{~K} 7$ \\
\hline
\end{tabular}

Figure 6. Switch Input Circuit 